\title{
Time Shift of Pulses Due To Dispersion Slope and Nonlinearity
}

\author{
Dietrich Marcuse, Fellow, IEEE, Curtis R. Menyuk, Fellow, IEEE, and Ronald Holzlöhner, Student Member, IEEE
}

\begin{abstract}
We show that the time delay of optical pulses traveling in long fibers is influenced by the dispersion slope and the fiber nonlinearity. Consequently, one or more new pulses that are inserted by add-drop operations into a pulse train that has already traveled a long distance may shift relative to the old pulses. This time shift delays the initial pulses more than the newly inserted ones, so that the newly inserted pulses can leave their time frames, leading to errors.
\end{abstract}

Index Terms - Error analysis, nonlinearities, optical fiber theory, optical propagation in nonlinear media, optical pulse injection, time-domain analysis..

\section{INTRODUCTION}

I N LONG fiber systems with optical amplifiers the nonlinear effect accumulates and becomes significant even at low peak pulse powers [1]. The Kerr nonlinearity causes broadening of the pulse spectrum as well as four-wave mixing among communication channels at different wavelengths.

An additional effect, not usually considered, is a nonlinear time shift of the pulses that is caused by the interaction of the Kerr nonlinearity with the dispersion slope. This nonlinear time shift is superimposed on the normal, linear time delay affecting all pulse propagation. In a train of pulses, all having essentially the same peak amplitude and traveling the same distance, the additional nonlinear time shift is not noticeable. However, it may become a problem in add-drop operations in which new pulses are added in midstream. The new arrivals would not yet have accumulated a broadened spectrum due to self-phase modulation and hence would suffer less nonlinear time delay than the old pulses, causing them to shift their positions relative to the old ones. This situation might occur in proposed OTDM networks [2] or even in future WDM networks in which several users might share a single transmission wavelength. A similar effect also occurs if pulses are injected at the same location but with different power as would occur for example in a duobinary transmission system [3].

Manuscript received May 13, 1999; revised September 2, 1999. This work was supported by grants from AFOSR, NSF, and DOE.

The authors are with the Department of Computer Science and Electrical Engineering, University of Maryland-Baltimore County, Baltimore, MD 21250 USA.

Publisher Item Identifier S 1041-1135(99)09481-1.

\section{THEORY}

As a measure of the time shift of a single pulse we use the first moment of the time variable $t$ defined as [4]

$$
\langle t(z)\rangle=\frac{\int_{-\infty}^{\infty} t|E(z, t)|^{2} d t}{\int_{-\infty}^{\infty}|E(z, t)|^{2} d t} .
$$

The variable $E(z, t)$ indicates the complex pulse envelope as a function of distance $z$ and time $t$. In terms of the pulse spectrum

$$
\psi(z, \omega)=\int_{-\infty}^{\infty} E(z, t) e^{i \omega t} d t
$$

the first temporal moment can also be expressed as

$$
\langle t(z)\rangle=\dot{\beta}_{0} z+\frac{\dddot{\beta}_{0} z}{2} \frac{\int_{-\infty}^{\infty}\left(\omega-\omega_{0}\right)^{2}|\psi(z, \omega)|^{2} d \omega}{\int_{-\infty}^{\infty}|\psi(z, \omega)|^{2} d \omega}
$$

where $\omega$ represents the angular frequency, $\beta_{0}$ the propagation constant of the optical wave at a central frequency $\omega_{0}$, and the dots signify differentiation with respect to $\omega$. A term with the second frequency derivative of $\beta_{0}$ is omitted because in most applications this term vanishes after it is averaged over the fiber length, and, in addition, it does not yield a contribution if the optical power spectrum is symmetrical around $\omega_{0}$.

Since $\dddot{\beta}_{0}>0$, the frequency integral in (3) makes a positive contribution to the normal group delay $\dot{\beta}_{0} z$. This contribution increases as the spectrum broadens due to nonlinear self-phase modulation.

Equation (3) for $\langle t(z)\rangle$ shows that the increase in the relative time delay is proportional to the distance $z$ traveled by the pulse if the power spectrum remains unchanged along the fiber, as is the case for linear or soliton transmission [1]. It is also true periodically for dispersion-managed solitons which are, therefore, exempt from a nonlinear time shift [5], [6]. However, when the spectrum broadens as a function of the length coordinate $z$, the additional time shift is no longer linear so that pulses that travel different distances or carry different amounts of power may be displaced with respect to each other. Finally, (3) also implies that the nonlinear time shift can be reduced if the fiber dispersion is arranged as a periodic dispersion map which decreases the tendency of the spectrum to spread with length [7]. 
We introduce the nonlinear time shift

$$
\langle\tau(z)\rangle=\langle t(z)\rangle-\dot{\beta}_{0} z .
$$

Using the nonlinear Schrödinger equation [8], [9] and approximating the pulse by its self-phase modulation solution applied to Gaussian-shaped pulses [10], the following approximate expression can be derived,

$$
\begin{aligned}
&\langle\tau(z)\rangle=\frac{\lambda^{4} \ln (2)}{\left(2 \pi c \tau_{h}\right)^{2}}\left(\frac{2 D}{\lambda}\right.\left.+\frac{d D}{d \lambda}\right) \\
& \cdot z\left\{1+\frac{1}{\sqrt{3}}\left(\frac{4 \pi}{3 \lambda} \frac{n_{2} P_{0}}{A_{\mathrm{eff}}} z\right)^{2}\right\} .
\end{aligned}
$$

The parameters appearing in this equation have the following meaning: The wavelength is $\lambda, c$ is the velocity of light in vacuum, $\tau_{h}$ is the full width of the pulse at half maximum, $D$ is the usual dispersion expressed as the wavelength derivative of the time delay, $n_{2}$ is the nonlinear coefficient, $P_{0}$ the peak power of the pulse, and $A_{\text {eff }}$ is the effective area of the guided fiber mode. The appearance of the group velocity dispersion, $D$, in this expression stems from the conversion of $\dddot{\beta}_{0}$ to the wavelength derivative of $D$.

Effectively, we are assuming that the dispersive contribution to the pulse spectrum and shape is small compared to the nonlinear contribution due to the self-phase modulation. Consequently, we neglect its contribution to these quantities in (5). However, we assume that it still shifts the pulse once the pulse's spectrum and shape are determined. As a consequence of this approximation, (5) is useful for estimating the magnitude of the time shift, but it is not always quantitatively accurate.

\section{SimUlations}

We now explore the nonlinear time shift in more detail with the help of numerical simulations of the nonlinear Schrödinger equation. To reduce the problem to its bare essentials, we consider a single input pulse with a raised cosine shape. This pulse occupies a time slot of $T=0.1 \mathrm{~ns}$, which corresponds to a bit rate of $10 \mathrm{~Gb} / \mathrm{s}$. In addition, we assume that the fiber is lossless. In a realistic fiber, losses would be compensated by periodically spaced amplifiers so that the peak power of pulses in the lossless system would have to be interpreted as a path-averaged power.

The relative time shift, $\tau$, of this pulse is expressed in two different ways. We compute its average value $\langle\tau\rangle$, according to (1) and (4) and also keep track of the temporal position of the peak of the pulse. Fig. 1 shows plots of these quantities for raised-cosine-shaped pulses with different peak powers and different dispersion histories. It also shows the theoretically expected time shift according to (5). The fixed parameters in both subplots have the following numerical values: $\lambda=$ $1.55 \mu \mathrm{m}, \tau_{h}=40 \mathrm{ps}, P_{0}=2 \mathrm{~mW}, A_{\mathrm{eff}}=55 \mu \mathrm{m}^{2}, n_{2}=$ $2.6 \times 10^{-20} \mathrm{~m}^{2} / \mathrm{W}$, and $d D / d \lambda=0.03 \mathrm{ps} / \mathrm{nm}^{2}-\mathrm{km}$. The average and peak time shifts can generally be quite different due to the distortion of the pulse under the combined influence of the nonlinearity and the dispersion slope. Fig. 1(a) was computed for a pulse with a peak power of $2 \mathrm{~mW}$ and no firstorder group velocity dispersion. In this case, the approximate
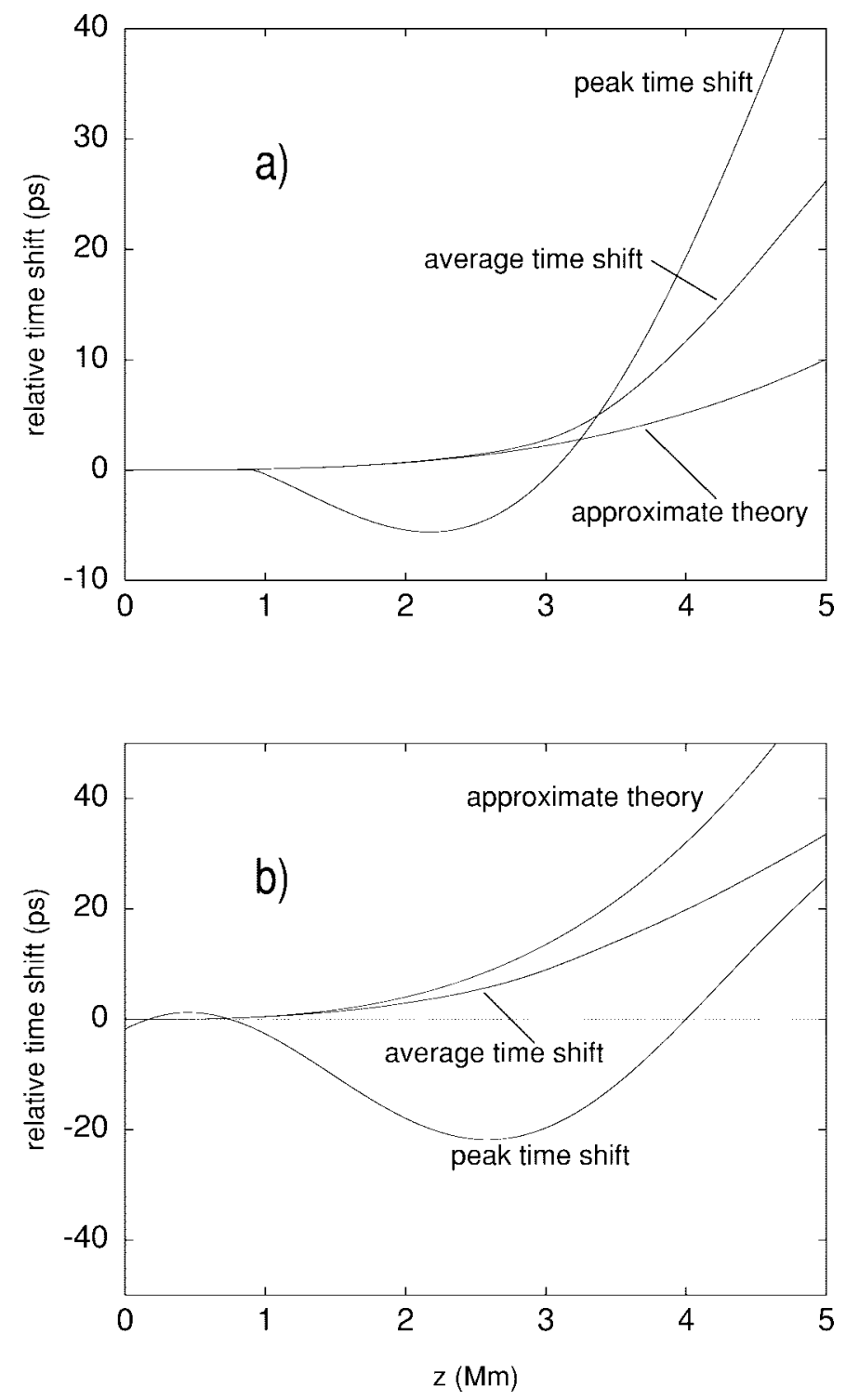

Fig. 1. Relative time shifts in picoseconds of a single pulse as a function of the length coordinate $z$ in megameters $(\mathrm{Mm})$ computed from the nonlinear Schrödinger equation and from the approximate theory. In (a), the peak pulse power was $2 \mathrm{~mW}$. There was no dispersion map and $D=0$. The full width of the pulse (FWHM) was $\tau_{h}=40 \mathrm{ps}$ at the input. In (b), the input peak pulse power was $5 \mathrm{~mW}$. There was a dispersion map with a $100 \mathrm{~km}$ period and an average normal dispersion of $\langle D\rangle=-0.01 \mathrm{ps} /(\mathrm{nm} \mathrm{km})$.

theory underestimates the average time shift because the pulse has narrowed and its peak height has increased. In Fig. 1(b), we raised the peak power of the incident pulse to $5 \mathrm{~mW}$ and also included a dispersion map with a period of $100 \mathrm{~km}$. The map consisted of three sections. The first and third sections had a normal dispersion of $D=-0.52 \mathrm{ps} / \mathrm{nm} \cdot \mathrm{km}$ and a length of $25 \mathrm{~km}$, while the middle section had an anomalous dispersion of $D=0.5 \mathrm{ps} / \mathrm{nm}-\mathrm{km}$ and a length of $50 \mathrm{~km}$. The slight average normal dispersion of $\langle D\rangle=-0.01 \mathrm{ps} / \mathrm{nm} \cdot \mathrm{km}$ was chosen to prevent the pulse from breaking up into solitons. In this case, the approximate theory yields higher time delays than the simulation because the dispersion map prevents the spectrum from spreading as rapidly as it would with $D=0$.

To demonstrate the time shift of a pulse that is injected midstream relative to the initial pulses, we generated a sequence of 

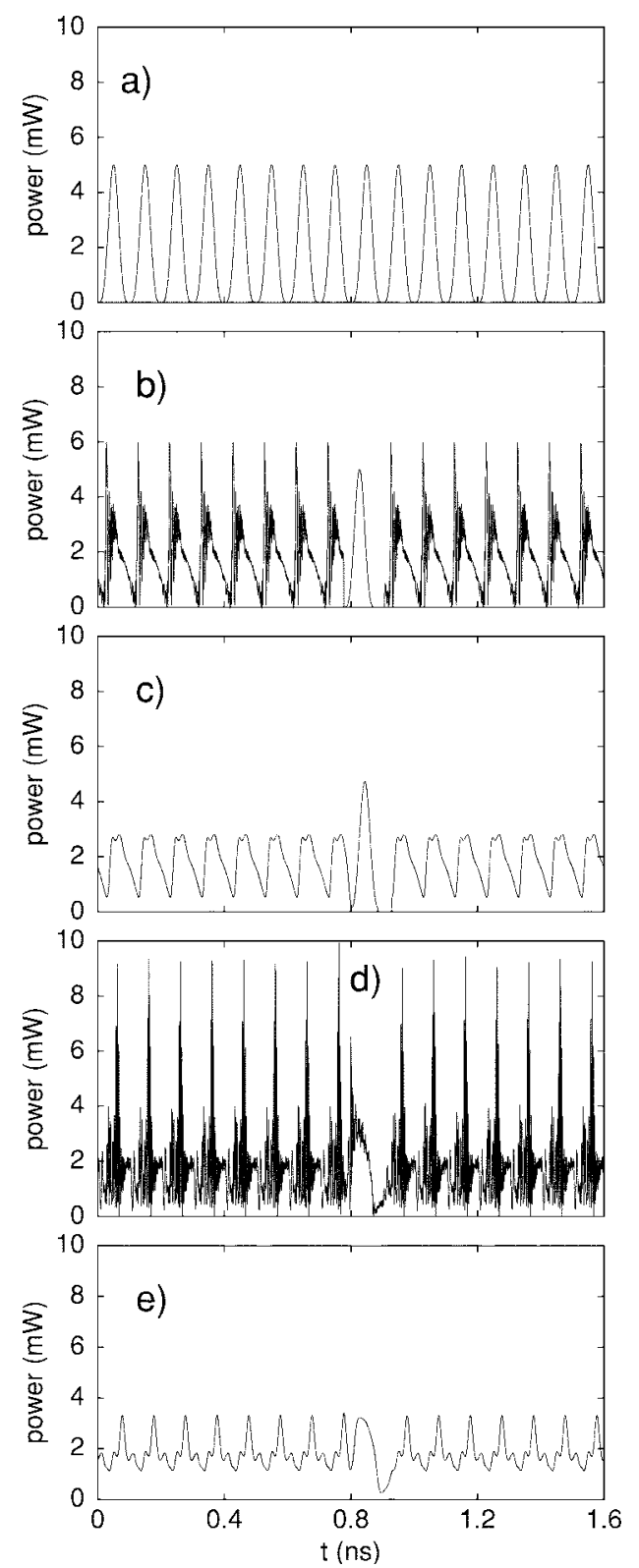

Fig. 2. In (a), we show the initial train of raised cosine input pulses at a bit rate of $10 \mathrm{~GB} / \mathrm{s}$. At $z=3 \mathrm{Mm}$, pulse no. 9 is replaced with a new pulse. In (b), we show the optical pulse train. Note the distortion of the old pulses caused by the relatively high peak pulse power. In (c) we show the pulse train of (b) after square-law detection and filtering with a baseband filter of $25 \mathrm{GHz}$. At $z=4.8 \mathrm{Mm}$ the new pulse no. 9 has shifted its position relative to the old pulses, as shown in (d). In (e), we show the pulse train of (d) after square-law detection and filtering with a baseband filter of $25 \mathrm{GHz}$.

16 raised cosine pulses with a peak value of $5 \mathrm{~mW}$, as shown in Fig. 2(a). The dispersion map was the same as that used in Fig. 1(b). After a distance of $3000 \mathrm{~km}$, we removed the 9th pulse and replace it with a new pulse with the same profile as the initial pulses had when we first injected them into the fiber. At this point, the optical profile of the original pulses is already distorted, and the peak positions of the pulses do not coincide with their average positions, as shown in Fig. 2(b). After detection and electrical filtering with a 4th order Bessel filter of $25-\mathrm{GHz}$ baseband bandwidth, the pulses assume the appearance shown in Fig. 2(c). A time shift is visible just from the action of the electrical filter. In Fig. 2(d), we show the optical profile after the pulses have propagated an additional $1800 \mathrm{~km}$. The peak position of the injected pulse has shifted by about 60 ps. The peak pulse curve of Fig. 1(b) indicates a delay of $55 \mathrm{ps}$ which is in reasonably good agreement. After electrical filtering, the pulses have the form shown in Fig. 2(e). The injected pulse has moved into the time slot of its neighbor so that it might be misidentified by the detection equipment.

We note that the time shift scales as the square of the power. Thus, it will become more important at higher data rates in which higher powers must be used to maintain a fixed signalto-noise ratio. Conversely, since bits are spaced closer together as the data rate increases, the time delay that can be tolerated diminishes. We conclude that this time shift effect will become even more important in systems that operate at $40 \mathrm{~Gb} / \mathrm{s}$ instead of $10 \mathrm{~Gb} / \mathrm{s}$.

\section{ACKNOWLEDGMENT}

C. R. Menyuk gratefully acknowledges encouragement from Dr. H. Mandelberg.

\section{REFERENCES}

[1] G. P. Agrawal, Nonlinear Fiber Optics. San Diego: Academic, 1995.

[2] R. A. Barry, V. W. S. Chan, K. L. Hall, E. S. Kintzer, J. D. Moores, K. A. Rauschenbach, E. A. Swanson, L. E. Adams, C. R. Doerr, S. G. Finn, H. A. Haus, E. P. Ippen, W. S. Wong, and M. Haner, "All-optical network consortium-Ultrafast TDM networks," IEEE J. Select. Areas Commun., vol. 14, pp. 999-1013, 1996.

[3] G. May, A. Solheim, and J. Conradi, "Extended $10 \mathrm{~Gb} / \mathrm{s}$ fiber transmission distance at $1538 \mathrm{~nm}$ using a duobinary receiver," IEEE Photon. Technol. Lett., vol. 6, pp. 648-650, 1994.

[4] D. Marcuse, "RMS width of pulses in nonlinear dispersive fibers," $J$. Lightwave Technol., vol. 10, pp. 17-21, 1992.

[5] V. S. Grigoryan, T. Yu, E. A. Golovchenko, C. R. Menyuk, and A. N. Pilipetskii, "Dispersion-managed soliton dynamics," Opt. Lett., vol. 22, pp. 1609-1611, 1997.

[6] N. J. Smith, N. J. Doran, W. Forysiak, and F. M. Knox, "Soliton transmission using periodic dispersion compensation," J. Lightwave Technol., vol. 15, pp. 1808-1822, 1997.

[7] I. Gabitov, E. G. Shapiro, and S. K. Turitsyn, "Optical pulse dynamics in fiber links with dispersion compensation," Opt. Commun., vol. 134, pp. 317-329, 1997

[8] C. R. Menyuk, "Nonlinear pulse propagation in birefringent optical fibers," IEEE J. Quantum Electron., vol. 23, pp. 174-176, 1987.

[9] __ "Pulse propagation in an elliptically birefringent Kerr medium," IEEE J. Quantum Electron., vol. 25, pp. 2674-2682, 1989.

[10] D. Anderson, "Variational approach to nonlinear pulse propagation in optical fibers," Phys. Rev. A, vol. 27, pp. 3135-3145, 1983. 